Ann. Parasitol. Hum. Comp., 1990, 65, Suppl. I : 5-10.
Mots-clés : Trypanosomes. Variation antigénique. Gènes d'antigènes. VSG. Procycline. Réarrangements du DNA. Transcription télomérique. Conversion génique.

Key-words: Antigenic variation. Coding genes. VSG. Procyclin. DNA organisation. Telomeric transcription. Gene conversion.

\title{
VARIATION ANTIGÉNIQUE DES TRYPANOSOMES AFRICAINS
}

\author{
E. PAYS
}

\begin{abstract}
RÉSUMÉ
Le génome des trypanosomes africains abrite une collection de plusieurs centaines de gènes d'antigènes variables différents, dont beaucoup sont localisés dans de petits chromosomes qui ne semblent servir qu'à cet effet. Un seul de ces gènes est exprimé à tout moment. Cette expression ne s'effectue que dans un de quelques 5 à 20 télomères compétents, et elle dépend de l'activité d'une RNA polymérase particulière, totalement insensible à l' $\alpha$-amanitine. La transcription du gène d'antigène n'est que l'aboutissement de celle d'une longue unité comprenant plusieurs gènes, dont l'un semble coder pour une adénylate cyclase. Le promoteur de transcription est actif de manière constitutive tout au long du cycle
\end{abstract}

parasitaire, même dans l'insecte vecteur où l'antigène variable n'est pas synthétisé. Ce paradoxe s'explique par l'existence de contrôles agissant au niveau de la progression de la RNA polymérase et au niveau de la stabilité des transcrits. Dans le sang du mammifère, le changement de manteau antigénique est dû à deux types de mécanismes : l'activation alternative des différents sites d'expression potentiels, ou des recombinaisons (principalement la conversion génique) entre le gène d'antigène actif et d'autres gènes du répertoire. Ces différents mécanismes permettent une certaine programmation dans l'ordre d'expression des antigènes.

\section{Summary : Antigenic variation in African trypanosomes.}

Although several hundred of different antigen genes exist in the trypanosome genome, only one is usually expressed at a time. This expression occurs in one of several possible telomeric expression sites. Besides being exclusively telomeric, transcription of the antigen gene exhibits other particular characteristics: the RNA polymerase is highly resistant to $\alpha$-amanitin, and the transcription unit comprises several other genes, one of which may encode an adeny- late cyclase. Post-transcriptional controls modulate the activity of this transcription unit during the parasite life-cycle. Antigenic variation is achieved through either alternative activation of different expression sites, or gene recombination within a given expression site. These mechanisms ensure a relative programming of antigen expression.

\section{INTRODUCTION}

Les trypanosomes africains, tels que Trypanosoma brucei, sont véhiculés par des glossines («mouche tsé-tsé ») qui les inoculent à divers mammifères lors de repas sanguins. Les trypanosomes présents dans les glandes salivaires de la mouche (forme "métacyclique ») sont préparés à ce nouvel environnement, et prolifèrent aussitôt dans le sang (forme « sanguicole »). Cette prolifération peut se poursuivre pendant de très longues périodes, en dépit de la réponse immunitaire de l'hôte, ce qui permet l'infestation d'autres glossines et le bouclage du cycle. Dans l'intestin moyen de l'insecte, le trypanosome se différencie en forme " procyclique », et se multiplie jusqu'à la maturation finale en forme métacyclique. Les différentes formes du parasite se caractérisent notamment par leur recouvrement de surface. Ainsi que le résume la figure 1, les trypanosomes

Département de Biologie Moléculaire, Université Libre de Bruxelles, 67, rue des Chevaux, B1640 Rhode Saint-Genèse, Belgique. métacycliques et sanguicoles expriment une protéine de surface appelée VSG (pour Variant Surface Glycoprotein), qui est remplacée par la procycline chez les trypanosomes procycliques. Ces deux protéines ont une structure très différente, mais toutes deux sont ancrées à la membrane plasmique par un radical glycophospholipidique, et toutes deux forment un manteau de surface homogène et dense (1). Alors que la fonction de la procycline est incomprise, il est clair qu'au moins un des rôles du VSG est d'assurer la défense du parasite contre le système immunitaire de l'hôte. En effet, cette molécule possède la double propriété d'être à la fois très immunogène et très variable, ce qui déroute continuellement la réponse immune. C'est à l'étude des mécanismes génétiques présidant à l'expression de cet antigène qu'est consacré cet article (pour plus de détails, voir 2,3 ).

\section{LES GÈNES DE VSG : STRUCTURE ET LOCALISATION}

Ainsi que schématisé dans la figure 2, les gènes de VSG se distinguent par la présence d'une région extrêmement variable codant pour les deux tiers N-terminaux de la pro- 


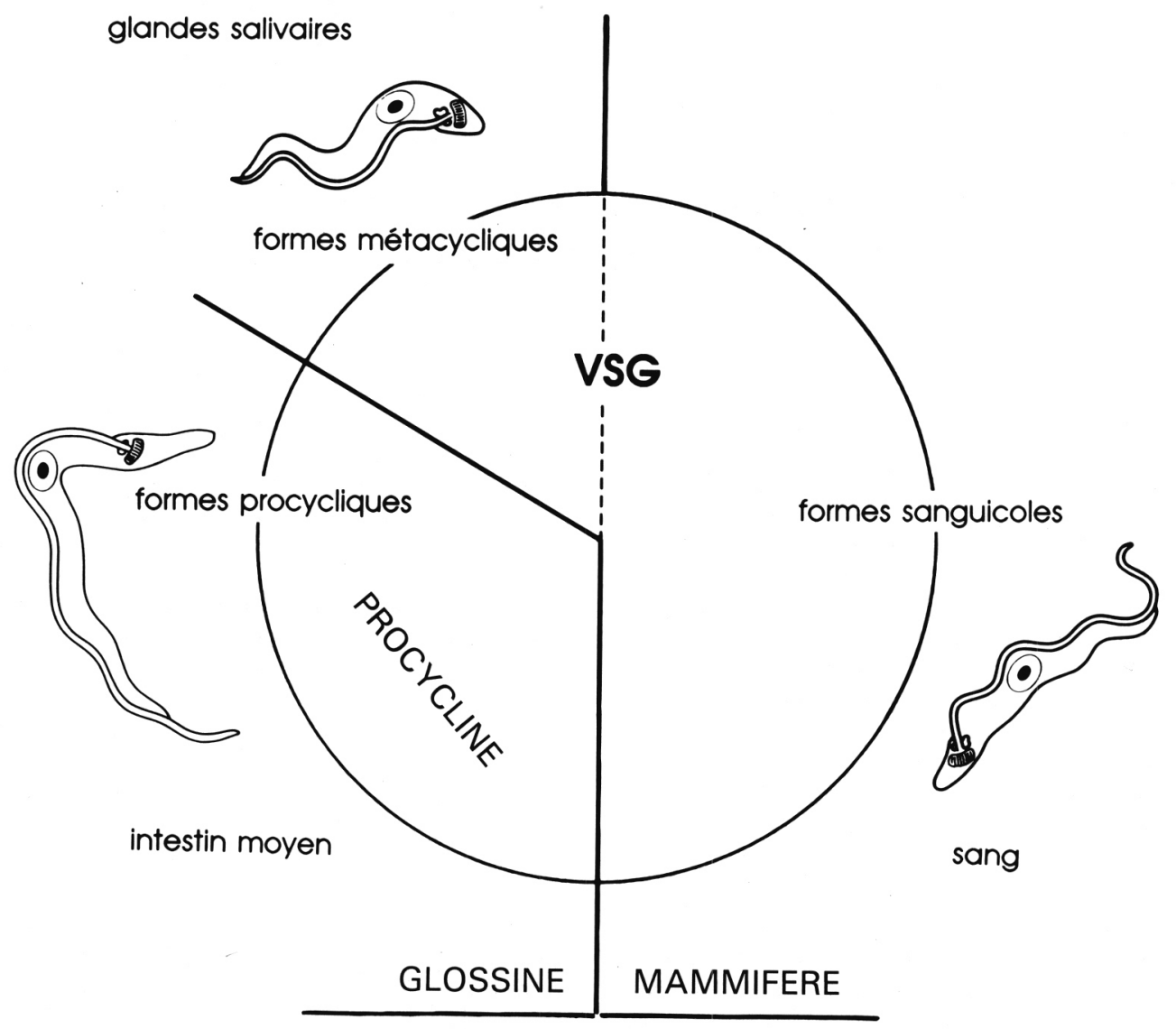

FIg. 1. - Protéines majeures de surface au cours du cycle parasitaire de Trypanosoma brucei.

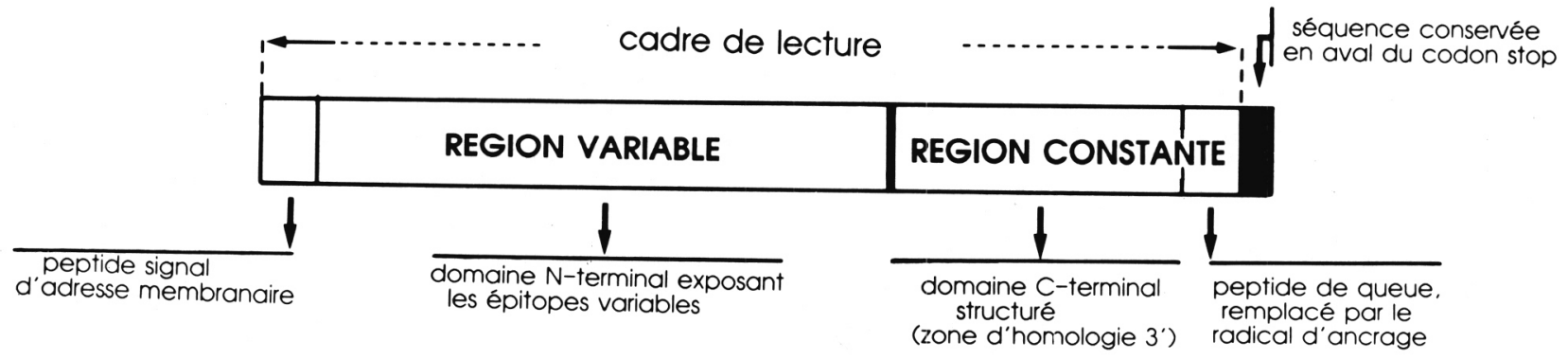

FIG. 2. - Structure générale des gènes de VSG.

téine, en amont d'une région conservée correspondant à un domaine C-terminal plus structuré. C'est grâce à des sondes de DNA correspondant à ce domaine conservé (bloc d'homologie $3^{\prime}$ ) que l'on a pu estimer le nombre total de gènes. Cette estimation oscille autour de mille copies. Il faut toutefois se garder d'utiliser ces données pour évaluer la taille du répertoire d'antigènes, car elles ne tiennent pas compte de l'existence de gènes non fonctionnels ou de gènes dupliqués; d'autre part, les mécanismes de réarrangements de séquences dont il sera question plus loin assurent un potentiel quasi illimité d'expansion du répertoire.
En fonction de leur environnement génomique, les gènes de VSG peuvent être répartis en deux catégories, les gènes télomériques et les gènes non télomériques (fig. $3 \mathrm{~A}$ et $\mathrm{B}$ ).

Beaucoup sont localisés en bout de chromosome, et sont donc télomériques. Généralement, c'est l'extrémité $3^{\prime}$ des gènes qui pointe vers le télomère. Les gènes télomériques sont flanqués de deux régions étendues de DNA simple (régions «barren »). La région "barren " 3 ' se compose de motifs typiquement télomériques, en particulier l'hexanucléotide TTAGGG qui est aussi retrouvé à l'extrémité des chromosomes humains. La région «barren » 5' est 


\section{(A) GENES TELOMERIQUES}

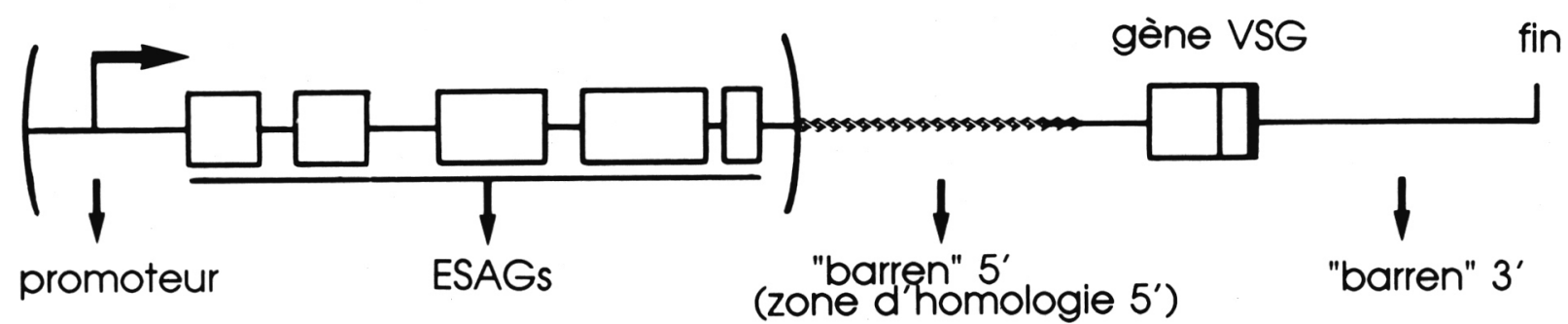

(B) GENES NON TELOMERIQUES

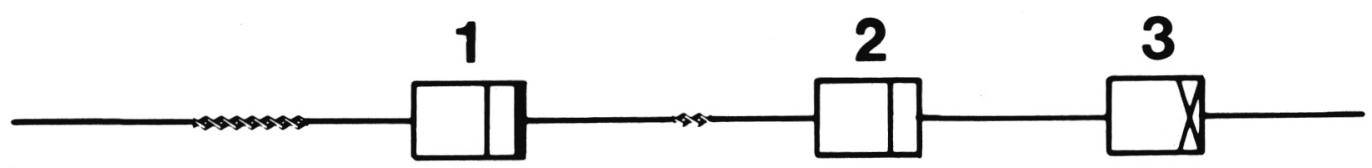

FIG. 3. - Organisation chromosomique des gènes de VSG. Le schéma en A symbolise l'environnement d'un gène télomérique. Ce gène est localisé à 5-10 kilobases de la fin d'un chromosome, et est flanqué de deux régions de DNA simple et répété (régions «barren »). La région en amont de la « barren " 5' peut, ou non (parenthèses), contenir d'autres gènes (ESAGs, pour « Expression Site-Associated Genes »), ainsi qu'un promoteur de transcription. Si ces séquences sont présentes, l'ensemble du télomère, d'une longueur de 50 à 70 kilobases, constitue un site d'expression potentiel pour gène de VSG.

En $B$ est représenté un modèle symbolique d'alignement de gènes non télomériques. Le gène 1 est flanqué des zones d'homologie requises pour recombiner avec le site d'expression (zigzags pour les répétitions $5^{\prime}$, boîte noire pour la zone conservée $3^{\prime}$ ), le gène 2 possède des blocs d'homologie insuffisants, et le gène 3 est doublement non fonctionnel, car il ne possède pas d'homologie 5' et son cadre de lecture est interrompu par des codons stops.

Aussi bien en A qu'en B, les éléments figurés ne sont pas à l'échelle.

constituée par la répétition, sur plusieurs kilobases, d'un motif d'environ 70 nucléotides particulièrement recombinogène. Cette région sert de bloc d'homologie 5' dans les mécanismes recombinatoires décrits plus loin. En amont de cette région, deux situations contrastées peuvent se présenter. Si le gène est localisé à l'extrémité de l'un des nombreux minichromosomes d'environ 50 à 150 kilobases qui caractérisent $T$. brucei, il semble que la région " barren " $5^{\prime}$ se prolonge en amont sans rencontrer d'autres types de séquences. En effet, mis à part certains éléments transposables, aucun autre gène que ceux de VSG n'a pu être localisé dans les minichromosomes. Il semblerait donc que ces derniers ne servent que de réservoirs à gènes de VSG. L'autre situation est celle d'un gène de VSG localisé dans un des sites d'expression potentiels. Dans ce cas, ainsi que l'indique la figure $3 \mathrm{~A}$ (région entre parenthèses), d'autres gènes sont présents immédiatement en amont de la région «barren " 5'. Nous allons y revenir dans la prochaine section.

Si de nombreux gènes de VSG sont télomériques, la plupart sont groupés plus à l'intérieur des chromosomes. La figure $3 B$ illustre différents exemples de gènes non télomériques. Dans l'exemple 1, les blocs d'homologie 5' et 3' sont tous deux présents. Ces blocs sont constitués des mêmes éléments que dans le cas des gènes télomériques, mais la région « barren " 5' est toujours moins étendue. Le nombre de motifs de 70 nucléotides varie d'un gène à l'autre : il peut être réduit (exemple 2) ou absent (exemple 3). De même, la zone d'homologie 3 ' peut être amputée ou non fonctionnelle par suite de mutations. Il est fréquent que les gènes non télomériques fassent partie de familles de séquences apparentées, dont certains membres peuvent coder pour des antigènes différents ou être non fonctionnels.

\section{EXPRESSION DES GÈNES DE VSG}

En dépit de l'étendue du répertoire, normalement un seul gène de VSG est exprimé à tout moment. Ceci semble destiné à assurer la formation d'un manteau antigénique homogène : dans les cas exceptionnels où deux antigènes sont synthétisés en même temps, le trypanosome choisit rapidement l'un des deux. Il se pourrait que des associations hétérogènes de VSG ne protègent pas efficacement le parasite contre l'action lytique du sérum, même non immun.

Le gène de VSG est transcrit dans un site d'expression télomérique. Les raisons de cette localisation particulière sont totalement incomprises. Différents sites d'expression possibles coexistent dans un même clone cellulaire; leur nombre n'est pas connu avec précision, mais se situe vraisemblablement entre 5 et 20 . La RNA polymérase qui transcrit le gène de VSG se caractérise par une résistance inhabituelle à l' $\alpha$-amanitine. Très curieusement, cette particularité est partagée par les gènes de procycline, en dépit de différences importantes dans la structure et l'acti- 
vité des deux types d'unités de transcription. Tout aussi curieusement, la transcription du gène de VSG est inversement corrélée à la manifestation d'une activité qui protège partiellement certains sites de restriction télomériques contre la digestion par les endonucléases spécifiques. Cette activité, encore non définie, pourrait résulter en une modification particulière et spécifique du DNA télomérique, qui ne s'opère donc qu'en cas d'inactivité du télomère. Le promoteur de transcription se situe loin en amont du gène de VSG, et d'autres gènes, appelés ESAGs pour Expression SiteAssociated Genes, font partie de la même unité de transcription (4) (fig. $3 \mathrm{~A}$ ). La fonction de ces gènes, ainsi que la signification de leur expression conjointe avec le gène de VSG, ne peuvent qu'être conjecturées à l'heure actuelle. Le seul indice existant est une homologie de séquence significative entre le gène ESAG 4 et les gènes connus d'adénylate cyclase eucaryotique, ce qui peut être mis en relation avec la découverte récente, chez $T$. brucei, d'une activité d'adénylate cyclase typiquement sanguicole. Cependant, la relation entre adénylate cyclase et VSG est loin d'être claire; le seul lien établi à ce jour est une stimulation spécifique de l'enzyme par des concentrations de calcium qui provoquent aussi le détachement du VSG. D'autre part, le gène ESAG 4, comme certains autres ESAGs, fait partie d'une famille multigénique dont d'autres membres sont exprimés à différents stades du cycle parasitaire, ce qui montre que des gènes semblables peuvent être exprimés sous des contrôles différents. Il semble que la transcription en une unité comprenant plusieurs gènes ne soit pas un caractère propre au site d'expression de gène VSG, mais commun à la plupart des gènes connus des trypanosomes. Ce phénomène est peut-être à relier au mécanisme particulier d'épissage, en trans, des longs transcrits primaires (2).

\section{Contrôles DE L'EXPRESSION DU GÈNE DE VSG}

La transcription du site d'expression subit au moins trois types de contrôles.

Dans le sang du mammifère, un mécanisme encore incompris assure la sélectivité de l'activité d'un seul site d'expression par cellule. Cependant, quoique exceptionnellement, deux sites peuvent être actifs simultanément, ce qui démontre que plusieurs promoteurs de transcription de gènes de VSG coexistent dans le génome. Il est donc clair qu'au stade sanguicole un mécanisme contrôle et sélectionne l'activité d'un promoteur de transcription parmi plusieurs candidats potentiels. C'est probablement le même mécanisme qui intervient dans l'activation alternative de différents sites d'expression au cours de la prolifération du parasite. Les spéculations actuelles au sujet de ce mécanisme d'exclusion font intervenir de possibles interactions entre le télomère actif et la matrice nucléaire, ce que la modification du DNA télomérique, uniquement observée en cas d'inactivité du gène, pourrait empêcher (5).

Un autre type de contrôle de l'activité du site d'expression s'opère au cours du cycle parasitaire. Lorsque la forme sanguicole se différencie en forme procyclique, soit par passage dans la glossine, soit par culture à basse température in vitro, la transcription du gène de VSG s'arrête très rapidement. Cette inactivation va de pair avec la stimulation immédiate de la synthèse du RNA messager de procycline, et donc le remplacement du VSG par la procycline à la surface du parasite. De manière frappante, le contrôle de l'activité de ces deux unités de transcription s'avère similaire, tout en opérant de façon inverse : dans les deux cas, l'environnement adverse du parasite conduit à un blocage rapide de l'élongation de la transcription, couplé à l'accélération de la dégradation des transcrits. L'activité des promoteurs de transcription respectifs ne semble cependant pas affectée (6). Ce type de contrôle, agissant essentiellement au niveau de la stabilité des transcrits, pourrait également assurer la différenciation d'autres fonctions cellulaires au cours du cycle parasitaire (7). Les facteurs intervenant dans ce processus ne sont pas connus, mais pourraient dépendre d'une synthèse protéique (8).

Un dernier type de contrôle s'observe dans les glandes salivaires de la mouche. A ce stade, l'activité de sites d'expression de gènes de VSG reprend. Toutefois, ce phénomène se distingue totalement de ce l'on observe dans la forme sanguicole. Tout d'abord, il ne concerne pas le site d'expression actif juste avant le passage du parasite dans la mouche. Ce dernier ne se réactive en effet qu'après l'injection du trypanosome dans le circuit sanguin du mammifère, sans doute par simple levée du bloquage de l'élongation de la transcription (9). Ensuite, ce phénomène est manifestement induit par un facteur de la mouche, qui déclenche simultanément, chez tous les trypanosomes arrivés à ce stade du cycle, l'activation de l'un des 10-20 sites d'expression spécifiquement «métacycliques ». Enfin, l'activité de ces sites s'éteint progressivement lorsque les trypanosomes sont injectés dans le sang, ce qui démontre que cette activité ne dépend pas de facteurs présents dans le sang. Les sites où cette expression particulière se produit semblent propres aux télomères des plus grands chromosomes. Ils pourraient différer des sites sanguicoles, par l'absence de la région "barren " 5' et des ESAGs. Cet isolement génétique par rapport aux sites d'expression sanguicoles pourrait être responsable de la conservation relative du répertoire antigénique métacyclique, qui se limite à un peu plus d'une dizaine de types différents. Toutefois, les types antigéniques métacycliques peuvent aussi être réexprimés plus tard, au cours de l'infection chronique dans le sang; cette réexpression passe très probablement par des mécanismes d'activation génique typiquement sanguicoles, que nous allons décrire dans la section suivante.

\section{MÉcanismes DE VARIATION DES GÈNES DE VSG}

La variation antigénique des trypanosomes sanguicoles résulte du fonctionnement de deux mécanismes fondamentalement différents : le changement de site d'expression et 


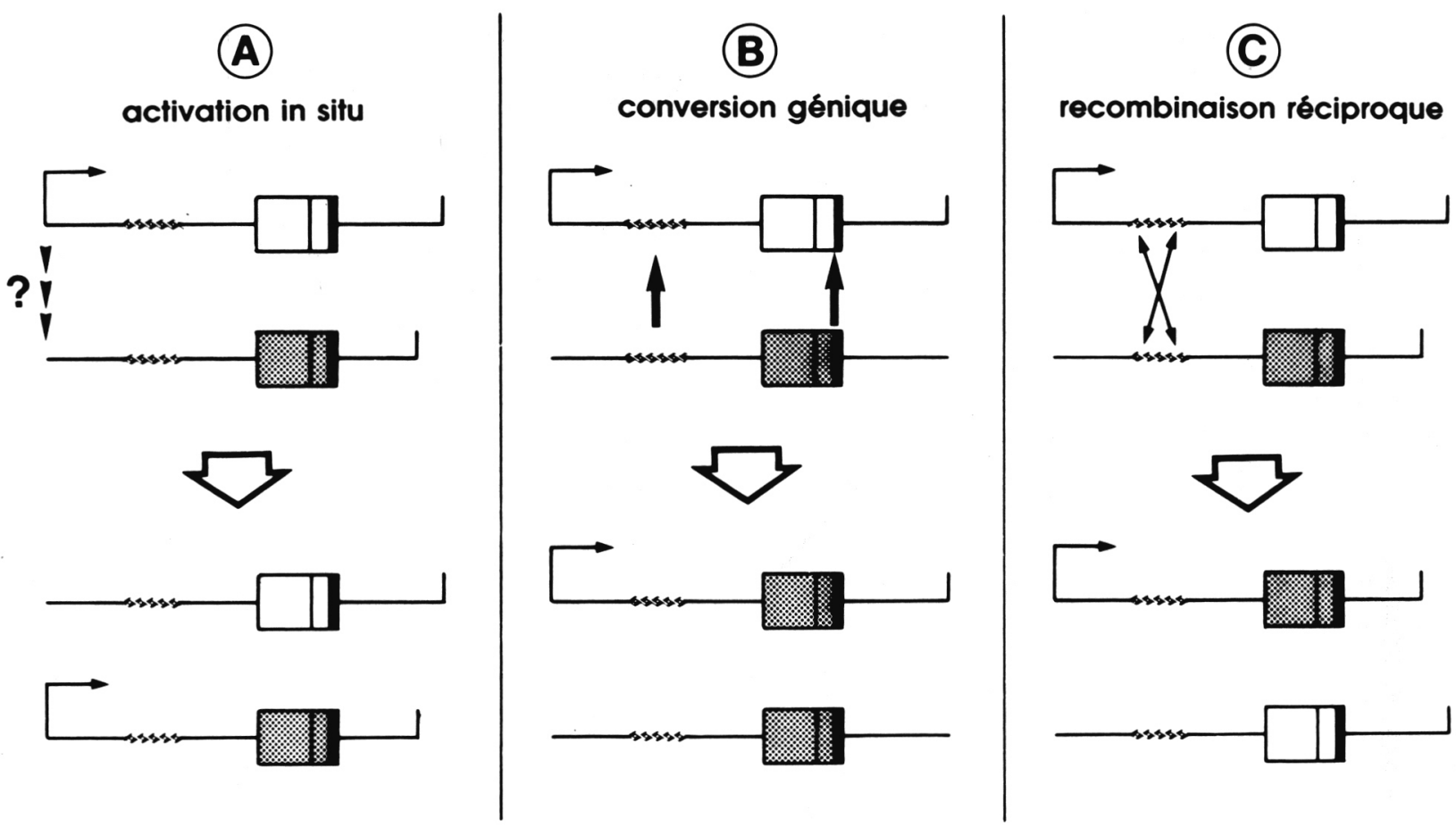

FIg. 4. - Mécanismes de variation antigénique. La flèche horizontale symbolise la transcription et définit le site d'expression actif.

le changement de gène de VSG au sein d'un même site d'expression (fig. 4).

Le premier mécanisme, baptisé " activation in situ », a déjà été évoqué plus haut. Il consiste en l'utilisation alternée de différents sites d'expression, sans réarrangement apparent du DNA, ni au niveau du gène de VSG, ni au niveau du promoteur de transcription. Étant donné que les différents sites d'expression potentiels portent des gènes de VSG différents, cette alternance entraîne un changement de type antigénique. Ce mécanisme s'observe principalement en début d'infection sanguicole, probablement parce qu'à ce stade aucune réponse immunitaire anti-VSG ne fait obstacle à la forte propension d'au moins plusieurs sites d'expression à s'activer spontanément.

Le second mécanisme regroupe différents types de recombinaison du DNA, qui permettent un remplacement, total ou partiel, du gène de VSG actif par un autre. Ce remplacement peut être un échange par recombinaison réciproque entre télomères, bien que ceci n'ait été observé que très rarement. Le plus souvent, le remplacement s'opère par conversion génique, c'est-à-dire par recombinaison non réciproque. Dans ce cas, le gène est remplacé par la copie d'un autre gène (voir réf. 10 pour plus de détails). Ce mécanisme recombinatoire est très fréquent entre membres de familles multigéniques. Chez le trypanosome, il présente la caractéristique d'être particulièrement flexible : les étendues de conversion génique peuvent aller de quelques nucléotides à plusieurs dizaines de kilobases. Le plus fréquemment, cette étendue va du bloc d'homologie $5^{\prime}$ au bloc 3', c'est-à-dire des motifs répétés de 70 nucléotides, généralement situés environ 1,5 kilobase en amont du gène, jusqu'à la région conservée en fin du gène. Lorsque la conversion se termine à l'intérieur d'un gène, tel qu'on l'observe tard en cours d'infection (voir ci-dessous), l'événement recombinatoire conduit à la formation de gènes composites, ce qui peut étendre le répertoire de gènes. Selon des estimations récentes, la fréquence des conversions géniques entre gènes de VSG serait particulièrement élevée. Ceci pourrait être attribué à la présence des régions " barren " : la région $5^{\prime}$ est un lieu privilégié de recombinaison, probablement par sa répétition et par la séquence particulière des motifs, et la région $3^{\prime}$, c'est-à-dire le télomère proprement dit, semble, comme dans les autres types cellulaires, être le siège d'interactions entre chromosomes, ce qui favorise aussi les recombinaisons (11).

\section{Programmation DE LA VARIATION ANTIGÉNIQUE}

Le répertoire de types antigéniques ne s'exprime pas totalement au hasard. Certains antigènes sont typiquement précoces, tandis que d'autres ne s'observent que rarement, ou exceptionnellement. Il est clair que cette programmation permet au parasite de prolonger l'infection, en évitant un épuisement trop rapide du leurre antigénique. Ce phénomène ne semble pas dépendre de la nature des antigènes ou de leurs gènes, mais plutôt de l'environnement génomique de ces derniers (12). Lorsqu'ils sont situés dans des 
sites d'expression potentiels, les gènes de VSG ont une forte probabilité d'expression, et il n'est donc pas étonnant que les gènes s'exprimant précocement soient télomériques. De même, les gènes télomériques non situés dans des sites d'expression, comme le sont ceux présents dans les minichromosomes, apparaissent facilement activables par recombinaison avec le site d'expression, probablement grâce au niveau élevé d'homologie partagé entre télomères. Ensuite peuvent s'exprimer les gènes non télomériques, qui ne peuvent avoir accès au site d'expression qu'en tant que matrices pour la synthèse d'une copie, c'est-à-dire en tant que donneurs dans des événements de conversion génique. Il existe toute une gamme de possibilités d'expression en ce qui concerne ces gènes. S'ils sont flanqués des deux blocs d'homologie nécessaires pour la recombinaison avec le site d'expression (gène 1 dans la fig. $3 \mathrm{~B}$ ), une conversion complète est possible d'un bloc à l'autre. Dans ce cas, il semble que la probabilité d'expression du gène dépende de l'ampleur des homologies. Parfois, le bloc d'homologie 5' peut être insuffisant (gène 2) ou même absent (gène 3), et le bloc d'homologie 3' peut être tronqué ou interrompu par des codons stop. Dans ces différents cas, le gène ne peut être exprimé facilement, soit parce que les homologies sont insuffisantes pour permettre la recombinaison avec le site d'expression, soit parce que le gène ne peut coder pour un antigène fonctionnel. Une solution peut se présenter lorsque le gène actif, présent dans le site d'expression, possède lui-même des homologies avec le donneur potentiel. Des recombinaisons dans ces zones d'homologie peuvent pallier les insuffisances, et générer des gènes fonctionnels. Ces événements recombinatoires sont nécessairement peu probables et tardifs, puisqu'ils dépendent de la nature du gène particulier présent dans le site d'expression. D'autre part, puisqu'ils se produisent au sein des gènes de VSG, ils conduisent à la formation de gènes composites. De tels gènes s'observent fréquemment tard dans l'infection chronique, et peuvent présenter une structure très complexe résultant de multiples événements recombinatoires, apparemment aléatoires. Il semble toutefois qu'en dehors des contraintes liées au changement des régions porteuses des épitopes antigéniques, des contraintes structurelles favorisent les recombinaisons aboutissant à l'échange des domaines $\mathrm{N}$ - et C-terminaux.

\section{CONCLUSION}

L'analyse génétique du phénomène de la variation antigénique des trypanosomes avait été entreprise, à l'origine, afin d'en comprendre les mécanismes dans l'espoir de pouvoir les enrayer, et donc d'envisager des méthodes de lutte prophylactique contre les trypanosomiases. Si ces méca- nismes sont maintenant mieux compris, il est clair que l'objectif initial est toujours hors d'atteinte. Le potentiel de variation du déterminant antigénique majeur, le VSG, semble déjouer l'attaque du chercheur aussi bien que la réponse du système immunitaire. En revanche, le trypanosome ne s'est pas montré avare d'informations, souvent remarquables. Si nous connaissons mieux comment sont structurés nos télomères, comment nos gènes d'antigènes varient ou comment ces antigènes s'ancrent dans la membrane plasmique, c'est en grande partie grâce à ce parasite exotique que nous le devons.

Remerciements. - Je remercie le Professeur M. Steinert pour ses commentaires sur ce manuscrit. Les recherches du laboratoire sont financées par le Fonds de la Recherche Scientifique Médicale, et des contrats avec la CEE (TDS-M-23B), Solvay et Cie (Bruxelles) et l'OMS (UNDP/World Bank/WHO Special Programme for Research and Training in Tropical Diseases).

\section{RÉFÉRENCES}

1. Braun-Breton C., Pereira da Silva L. H. : Modulation de l'activité biologique des protéines de surface chez les protozoaires hémoparasites. Med. Sci., 1989, 5, 736-743.

2. Borst P. : Discontinuous transcription and antigenic variation in trypanosomes. Ann. Rev. Biochem., 1986, 55, 701-732.

3. Pays E., Steinert M. : Control of antigen gene expression in african trypanosomes. Ann. Rev. Genet., 1988, 22, 107-126.

4. Pays E., Tebabi P., Pays A., Coquelet H., Revelard P., Salmon D., Steinert M. : The genes and transcripts of an antigen gene expression site from T. brucei. Cell, 1989, 57, 835-845.

5. Greaves D. R., Borst P. : T. brucei variant-specific glycoprotein gene chromatin is sensitive to single-strand-specific endonuclease digestion. J. Mol. Biol., 1987, 197, 471-483.

6. Pays E., Coquelet H., Tebabi P., Pays A., Jefferies D., Steinert M., Koenig E., Williams R. O., Roditi I. : T. brucei: constitutive activity of the VSG and procyclin gene promoters. EMBO J., 1990 (sous presse).

7. Gibson W. C., Swinkels B. W., Borst P. : Post-transcriptional control of the differential expression of phosphoglycerate kinase genes in T. brucei. J. Mol. Biol., 1988, 201, 315-325.

8. Ehlers B., Czichos J., Overath P. : RNA turnover in T. brucei. Mol. Cell. Biol., 1987, 7, 1242-1249.

9. Pays E., Coquelet H., Pays A., Tebabi P., Steinert M. : T. brucei: post-transcriptional control of the variable surface glycoprotein gene expression site. Mol. Cell Biol., 1989, 9, 4018-4021.

10. Pays E. : DNA recombinations and transposition in trypanosomes. In: Kingsman A. J., Kingsman S. M., Chater K. F. (eds), Transposition. Cambridge University Press, Cambridge, 1988, 301-342.

11. Van der Werf A., Van Assel S., Aerts D., Steinert M., Pays E. : Telomere interactions may condition the programming of antigen expression in T. brucei. EMBO J., 1990, 9, 1035-1040.

12. Pays E. : Pseudogenes, chimaeric genes and the timing of antigen variation in African trypanosomes. Trends Genet., 1989, 5, 389-391. 